\section{Inherited metabolic disorders involving the eye: $a$ clinico-biochemical perspective}

\section{Abstract}

The diagnosis of inborn errors of metabolism is challenging for most physicians. Improvements in medical technology and greater knowledge of the human genome are resulting in significant changes in the diagnosis, classification, and treatment of inherited metabolic disorders (IMDs). Many known inborn errors of metabolism will be recognised earlier or treated differently because of these changes. It is important that physicians recognise the clinical signs of IMDs and know when to propose advanced laboratory testing or referral to a higher centre for better patient management. Ocular manifestations occur in various metabolic disorders. Although there is an extensive understanding of many inborn errors of metabolism at the biochemical, molecular, and metabolic levels, little is known about their pathogenesis. In particular, how systemic metabolic disease contributes to ocular defects remains to be elucidated in IMDs. The occurrence of eye abnormalities could be due to direct toxic mechanisms of abnormal metabolic products or accumulation of normal metabolites by errors of synthetic pathways or by deficient energy metabolism. A detailed ophthalmological assessment is essential. Definitive diagnosis and management of patients with IMDs is ideally carried out by a combination of specialists, including an ophthalmologist, paediatrician, biochemist, and medical geneticist. Recent advances in the diagnosis and treatment of IMDs have substantially improved the prognosis for many of these conditions.

Eye (2010) 24, 507-518; doi:10.1038/eye.2009.229; published online 2 October 2009

Keywords: IMDs; galactosaemia; Wilson's disease; gyrate atrophy of the retina; screening and testing for IMDs
M Rajappa, A Goyal and J Kaur

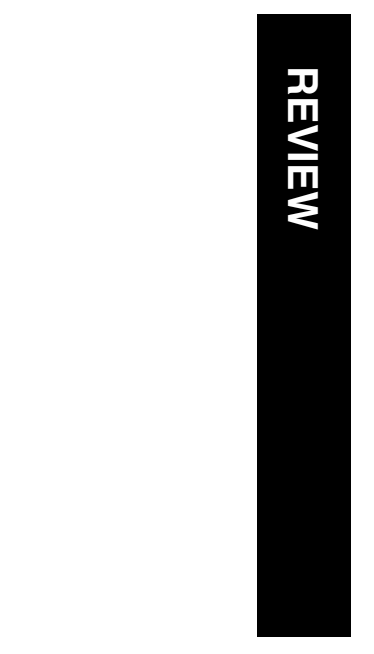

The field of inherited metabolic disorders (IMDs) is often thought to have been born in 1902 when Archibald Garrod published his first paper describing alkaptonuria and first coined the term 'inborn error of metabolism'. Although this discovery did occur in professional circles, it went largely unnoticed and fell into obscurity. ${ }^{1}$ Garrod recognised that some human disorders were due to enzyme deficiencies and these were because of genetic abnormalities inherited in an autosomal recessive manner. These insights were virtually ignored for 70 years and these ideas were brought to fruition by Barton Childs in $1970 .^{2}$

Inherited metabolic disorders are challenging for most physicians. The number of known IMDs is probably as large as the number of presenting symptoms that may indicate metabolic disturbances. ${ }^{3-5}$ Furthermore, physicians know they may not encounter certain rare IMDs during a lifetime of practice. There is an accelerating demographic switch from communicable diseases to genetic disorders. The expression of a genetic disease is the combined effect of genes and the environment. There are 25 million births in India annually; 0.8 million are born with congenital malformations; 0.35 million with glucose-6-phosphatase deficiency; 25000 with IMDs; 20000 with Down's syndrome, 15000 with congenital hypothyroidism; 14000 with thalassaemia; and 5000 with sickle cell anaemia. In India, biochemical screening of 4400 cases of mental retardation revealed that 5.75\% (256 cases) were due to various IMDs. ${ }^{6,7}$

Inborn metabolic disorders constitute a heterogeneous group of disorders affecting the metabolic pathways with an underlying genetic defect. IMDs are individually rare, but collectively common. IMDs are becoming increasingly recognised wherein early diagnosis and appropriate treatment interventions are
Department of Ocular Biochemistry, Dr Rajendra Prasad Centre for Ophthalmic Sciences, All India Institute of Medical Sciences, New Delhi, India

Correspondence: J Kaur, Department of Ocular Biochemistry, Dr Rajendra Prasad Centre for Ophthalmic Sciences, All India Institute of Medical Sciences, Ansari Nagar, New Delhi 110029, India Tel: +91 11 26593161; Fax: +911126588919 rediffmail.com

Received: 17 July 2008 Accepted in revised form: 6 August 2009 2009 E-mail: kaurjasbir@ Published online: 2 October 
mandatory to reduce the morbidity and mortality rates among the newborns. ${ }^{8}$ Early diagnosis is crucial for three reasons. ${ }^{6}$ First, IMDs are rapidly progressive and cause irreversible damage early in the course of the disease. Second, the treatment can often be effective, if commenced early and long-term outcome may be improved. Lastly, correct early diagnosis helps in genetic counselling.

The latest discoveries in the human genome project and advances in medical technology have resulted in significant alterations in the diagnosis, classification, and treatment of IMDs. These changes will aid in early recognition and treatment of many known IMDs. It is absolutely essential that doctors diagnose IMDs early and whenever necessary, refer the patient to a tertiary care centre for better care, detailed laboratory workup, and management. The latest breakthroughs in the diagnosis and treatment of IMDs have significantly improved the prognosis for many of these conditions. ${ }^{9}$

\section{Eye and IMDs}

The eye is the fourth most common system affected by genetic disease. ${ }^{10}$ More than 200 loci for genetic ocular diseases have been mapped so far $^{11,12}$ and the quest continues. Hereditary eye abnormalities can either manifest as primarily isolated disorders in which disease process in confined to the eye or as part of a systemic disease. The age of onset of ocular abnormalities in metabolic disease is variable, but onset often begins in childhood, infancy, or from birth. This paper discusses the major IMDs associated with eye abnormalities.

Ophthalmologic manifestations occur in various metabolic disorders. Although there is an extensive understanding of many inborn errors of metabolism at the metabolic, biochemical, and molecular levels, their exact pathogenesis remains to be established. The mechanisms by which systemic metabolic disease contributes to ocular defects remains to be elucidated. The various mechanisms involved could be due to direct toxic mechanisms of abnormal metabolic products, accumulation of normal metabolites by errors of synthetic pathways, or by deficient energy metabolism.

Single gene defects result in abnormalities in the synthesis or catabolism of proteins, carbohydrates, or fats (Table 1). Most are due to a defect in an enzyme or transport protein, which results in a block in a metabolic pathway (Figure 1). Effects are due to toxic accumulations of substrates before the block, intermediates from alternative metabolic pathways, and/ or defects in energy production and utilisation caused by a deficiency of products beyond the block. ${ }^{13}$ Every metabolic disease has several forms that vary in age of onset, clinical severity, and often, mode of inheritance.

Table 1 Classifications of inherited metabolic disorders ${ }^{13,14}$

\begin{tabular}{ll}
\hline A. Small molecule disorders & B. Organelle disorders \\
\hline $\begin{array}{l}\text { Carbohydrate metabolism } \\
\text { eg: Mucopolysaccharidoses, galactosaemia }\end{array}$ & $\begin{array}{l}\text { Lysosomal disorders } \\
\text { eg: Sphingolipidoses, glycoproteinoses, mucopolysaccharidoses, } \\
\text { gangliosidoses (Gaucher's disease, Niemann-Pick's disease, } \\
\text { Metachromatic leukodystrophy, Krabbe's disease) }\end{array}$ \\
$\begin{array}{l}\text { Protein metabolism } \\
\text { eg: Gyrate atrophy of the retina, cystinosis, Marfan's syndrome, } \\
\text { homocystinuria, Costeff's optic atrophy syndrome or type III 3- } \\
\text { methylglutaconic aciduria, urea cycle defects }\end{array}$ & $\begin{array}{l}\text { Mitochondrial disorders } \\
\text { eg: Defects in mitochondrial } \beta \text {-oxidation of fatty acids, Leigh's } \\
\text { disease, Alper's disease, citric acid cycle defects, pyruvate } \\
\text { dehydrogenase deficiency, Kearns-Sayre's syndrome, Leber's } \\
\text { hereditary optic neuropathy }\end{array}$ \\
$\begin{array}{l}\text { Lipid metabolism } \\
\text { eg: Fish eye disease, sphingolipidoses, Zellweger's syndrome } \\
\text { (peroxisome biogenesis disorders), Refsum's disease (deficiency } \\
\text { of phytanic acid oxidase), Bassen-Kornzweig's syndrome }\end{array}$ & $\begin{array}{l}\text { Peroxisomal disorders } \\
\text { eg: Zellweger's syndrome, pseudo-Zellweger's syndrome, } \\
\text { neonatal adrenoleukodystrophy, pseudo-neonatal } \\
\text { adrenoleukodystrophy, rhizomelic chondrodysplasia punctata, } \\
\text { hyperpipecolic acidaemia }\end{array}$
\end{tabular}

Nucleic acid metabolism eg: Lesch-Nyhan's syndrome

Porphyrin metabolism eg: Porphyrias

Metal metabolism eg: Wilson's disease, Menke's disease, haemochromatosis 
Environmental factors may trigger the onset and severity of disease. It also depends on degree of accumulation of toxic substances before metabolic block, for example, diet, intercurrent infection, fasting drugs, and so on.

Recognition of IMDs is important as it is quite common in incidence. The indications for metabolic studies to rule out the possibility of IMDs are discussed in Table 2. Early diagnosis is important, as in most cases, dietary restriction and early therapy prevents onset of disability. Prenatal diagnosis using amniocentesis and chorionic villus sampling may help to reduce the burden due to IMDs. Carrier testing is helpful and the possibility of partial defects or variants with normal activity must be remembered. The role of genetic counselling is invaluable in reducing the load due to IMDs and helps prevent high incidence in most cases. ${ }^{14}$

\section{Ocular manifestations of IMDs}

Either a patient presents with a known IMD and eye defect appears as a known manifestation of the disease, or the patient comes to the outpatient department primarily with ocular abnormalities and an IMD is suspected. ${ }^{15}$ Classical clinical approaches continue to

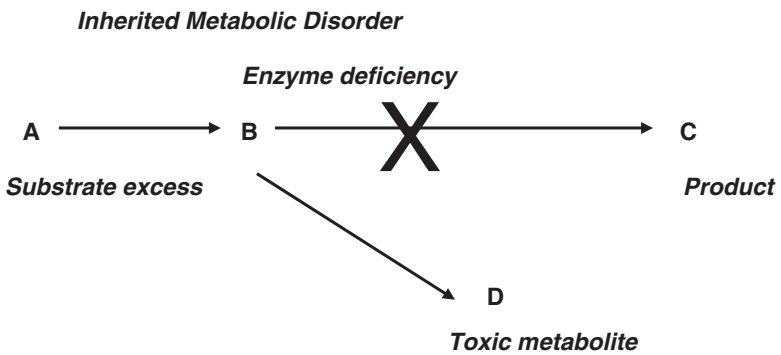

Figure 1 Garrod's Hypothesis. ${ }^{9}$ have a major role in diagnosis of patients with IMDs. Hereditary diseases of the eye have symmetrical bilateral involvement, as do those in metabolic disorders. Severe visual impairment is seen usually around 2 months of age, when eye contact develops in most children. In fact, poor vision may be defective in first few weeks of life. Anomalies of the eye are easily recognised as in cataracts in galactosaemia. In others as in peroxisomal diseases, fundoscopic examination may be normal in the neonatal period, whereas recordings of electroretinogram (ERG) and visual evoked responses are already abnormal. ${ }^{15}$

\section{IMDs involving the cornea}

The cornea may be affected directly or indirectly in many systemic diseases and by many different mechanisms leading to compromise of transparency, optical function, or structural integrity. Inherited disorders of metabolism of proteins, carbohydrates, or lipids can lead to accumulation of substances, which may become evident as opacities in the corneal epithelium (for example, Fabry's disease), stroma (for example, cystinosis), or Descemet's membrane (for example, Wilson's disease). Some disorders of protein formation may cause structural abnormalities of the cornea. Accumulation of a metabolic pathway product in the cornea due to enzyme deficiency or mutation of gene encoding the enzyme, leads to staining of the cornea in several IMDs.

The mucopolysaccharidoses (MPSs) are a heterogeneous group of disorders of errors in the carbohydrate metabolism with severe ocular involvement (corneal opacification, retinal degeneration, and optic atrophy) characterised by accumulation of glycosaminoglycans within multiple organ systems. ${ }^{16}$ Dermatan and keratan sulphate are deposited in the cornea and the degree of storage in keratinocytes

Table 2 Indications for metabolic studies ${ }^{9}$

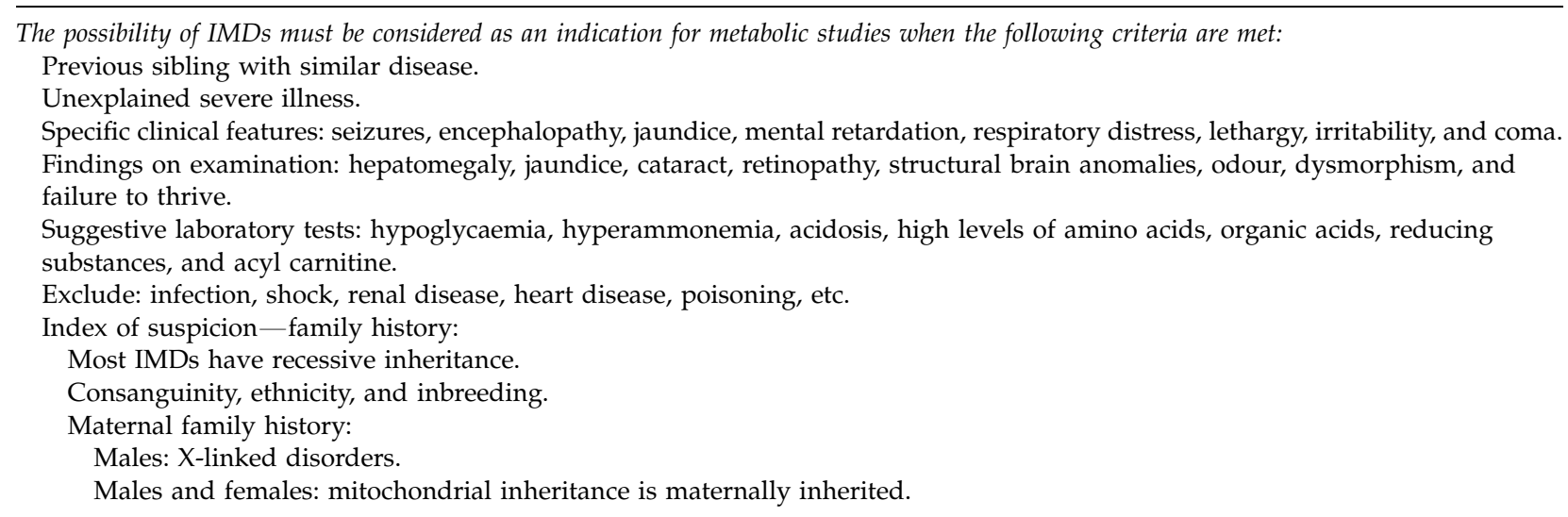


determines the degree of corneal clouding. ${ }^{15,16}$ Patients with MPS I (Hurler's syndrome and MPS VI (MaroteauxLamy syndrome) have corneal opacification, which can lead to difficulties in diagnosis and monitoring of glaucoma, optic disc changes, and retinopathy.

Fabry's disease is an X-linked glycosphingolipid storage disorder that is caused by the deficient activity of lysosomal $\alpha$-galactosidase $\mathrm{A}$, resulting in accumulation of glycolipids, mainly globotriaosylceramide, GL-3. Ocular findings are important in aiding diagnosis: engorged conjunctival vessels, congested retinal vessels, and corneal opacities. Enzyme-replacement therapy (ERT) should be given early to patients with Fabry's disease if resources allow before irreversible pathology sets in. Enzyme-enhancement therapy has also been shown to dramatically improve the cardiac condition of a case of cardiac variant of the disease. ${ }^{17}$

Another IMD in which recognition of corneal defect is important is Wilson's disease. Wilson's disease is an inborn error of copper metabolism, having an autosomal recessive inheritance with a mutation in the $A T P 7 B$ gene encoding a membrane bound copper-transporting ATPase. ${ }^{18}$ Its defect leads to increased copper deposition due to impaired biliary copper excretion. There is sunflower cataract and pigmented corneal rings called as Kayser-Fleischer (K-F) rings, due to copper deposit in the outer rim of the cornea.

Treatment for Wilson's disease is effective if diagnosis occurs before the onset of life-threatening symptoms. The goal of treatment is to remove excessive copper from the body and prevent it from re-accumulating. If treatment is stopped, the disease can be fatal. Drugs used for the initial treatment of Wilson's disease include penicillamine and trientine. ${ }^{19-21}$ These act by chelation or binding of copper, causing increased urinary excretion and rapidly reducing the copper content in the body. Tetrathiomolybdate is another chelating drug that will soon be approved by the FDA for initial treatment of Wilson's disease. ${ }^{21}$ For long-term care, zinc acetate is preferred, which acts by blocking the absorption of copper in the intestinal tract. This action depletes accumulated copper and prevents its re-accumulation. A major advantage of zinc therapy is its lack of serious side effects. In some cases, the condition is diagnosed at the stage of acute liver failure and involvement of the central nervous system (CNS). When this happens, a liver transplant is required in most cases. ${ }^{21}$

Fish eye disease with partial lecithin carnitine acyl transferase (LCAT) deficiency and low HDL cholesterol levels (due to mutation of LCAT gene) and familial hypercholesterolaemia (with mutation of LDL-receptor gene and hence elevated levels of LDL cholesterol) may lead to corneal opacities and hence, early detection is warranted to check further damage. Cystinosis is a rare autosomal recessive metabolic disorder in which nonprotein cystine accumulates within cellular lysosomes owing to a defect in lysosomal cystine transport. The pathognomonic ocular manifestation of cystinosis is the deposition of distinctive iridescent crystals in the cornea. ${ }^{22}$ Ocular symptoms include glare, photophobia, decreased corneal sensation, and mild decrease in vision.

\section{IMDs involving the lens}

Ocular manifestations are common in MPSs ${ }^{16,23}$ and may result in significant visual impairment. Corneal opacification, lens opacification, optic nerve swelling and atrophy, retinopathy, and glaucoma are some of the major lens manifestations of MPSs.

In galactosaemia, the deficiency of the enzyme (galactose-1-phosphate uridyl transferase) responsible for the breakdown of galactose, leads to build-up of galactose, which becomes toxic as the body makes toxic intermediates by an alternate pathway. The lens is avascular in nature, receiving its nutrients from aqueous humour. Most of the glucose metabolism is by anaerobic glycolysis. In absence of the enzyme (galactose-1phosphate uridyl transferase), galactose is reduced by aldose reductase to galactitol. Polyol accumulation causes cataract due to increase in intracellular fluid causing lens swelling increased membrane permeability and electrolyte abnormalities. ${ }^{24}$ Galactitol is toxic to the lens, causing opacification due to lens swelling shift of water into the lens and disruption of lens structure. Dietary restriction after screening is beneficial. Early identification and elimination of galactose from diet reverses growth failures, renal or liver dysfunction, and has better prognosis. Peroxisomal disorders are associated with cataract.

Cerebrotendinous xanthomatosis (CTX) is a rare inborn disorder of bile acid synthesis in which hepatic conversion of cholesterol to cholic and chenodeoxycholic acids (CDCAs) is impaired. ${ }^{25} \mathrm{~A}$ defect in hydroxylation of the cholesterol side chain that impairs oxidative cleavage has been identified. ${ }^{26}$ Thus, laboratory findings include elevated plasma levels of cholestanol and bile alcohols and increased urinary excretion of bile alcohol glucuronides with diminished biliary concentrations of CDCA. ${ }^{27}$ Cataracts, progressive neurological symptoms, and mild pulmonary insufficiency are unique features that distinguish CTX from other xanthomatous disorders. Treatment of CTX with CDCA, a bile acid, will arrest, but generally will not reverse the frequently devastating neurological deterioration seen in these patients. Xanthomatous tendon deposits have been reported to regress with CDCA therapy. Medical therapy, therefore, should be instituted at the time of diagnosis, and family members should be screened for subclinical disease. 
Treatments by oral administration of CDCA alone, 3-hydroxy-3-methylglutaryl CoA reductase inhibitor (pravastatin) alone, and combination of the two drugs have been attempted for patients with CTX. ${ }^{28}$ The combination of CDCA and pravastatin was a good treatment for CTX, based on the improvement of serum lipoprotein metabolism, the suppression of cholesterol synthesis, and reductions of cholestanol and plant sterol levels. In all patients, the progression of disease was arrested, but dramatic effects on clinical manifestations, xanthoma, and electrophysiological findings could not be found after the treatment of these drugs.

Another major consequence of IMDs is dislocation of the eye. Marfan's syndrome is an inherited disorder of the connective tissue. It is characterised by skeletal and cardiac abnormalities, aortic aneurysms, and ectopia lentis. The biochemical defect is a mutation on the fibrillin gene. It is inherited as an autosomal dominant trait. Lens subluxation is due to microfibril abnormalities of the lens capsule in Marfan's syndrome. ${ }^{29}$ Subluxation is bilateral, symmetrical, and upward. The dislocation may be complete, with the lens floating free within the vitreous cavity. Iridodonesis (tremulousness of the iris) may occur from non-support of the overlying iris by the lens. Axial length of the globe is increased leading to axial myopia. Retinal detachment is common. Glaucoma may be result of angle anomaly or may be associated with lens subluxation.

Another major cause for ectopia lentis is homocystinuria. ${ }^{30}$ Homocystinuria is a metabolic disorder due to deficiency of the enzyme cystathionine $\beta$-synthase producing increased urinary homocysteine and methionine. It has an autosomal recessive inheritance. The lens dislocation in homocystinuria commonly occurs inferiorly. ${ }^{30}$ Early detection by screening helps prevent complications by withholding methionine from diet and adding cysteine and large doses of vitamin $\mathrm{B}_{6}$. The zonular fibres are composed of glycoprotein with high concentration of sulphurcontaining amino acids, which explains their susceptibility to abnormal formation in IMDs of sulphur metabolism.

Ectopia lentis may occur in sulphite oxidase deficiency and molybdenum cofactor deficiency, ${ }^{31}$ leading to sulphocysteinuria as $S$-sulpho-L-cysteine accumulates in the lens. Lens membrane is rich in cholesterol, which helps in normal maintenance of the lens. Disorders of cholesterol biosynthesis may be associated with opacities of the crystalline lens. Ehler-Danlos syndrome is one of the inheritable connective tissue disorders due to defect in synthesis of collagen type I and III. Ehler-Danlos syndrome type VI manifests with ocular findings, such as keratoconus, fragility of the eye, and marfanoid habitus.

\section{IMDs involving the retina}

There are over 400 known inherited diseases in which the retina is substantially involved in the disease process. ${ }^{15,32}$ Retinitis pigmentosa (RP, prevalence 1 of 4000$)^{33}$ is a set of hereditary retinal dystrophies, characterised by pigment deposits in the fundus and progressive death of photoreceptors, always associated with the alteration of retinal pigment epithelium and retinal degeneration. Genetic heterogeneity of the typical non-syndromic form (rod-cone dystrophy) is extensive: eleven genes and one locus were reported for autosomal dominant $\mathrm{RP}$, seventeen genes and five loci for autosomal recessive $\mathrm{RP}$, and two genes and two loci for X-linked RP. ${ }^{34}$ Altogether, the two most frequently involved genes are RPGR (13\% of all RP cases) and $\mathrm{RHO}(4 \%)$, an important consideration for molecular diagnosis. ${ }^{34}$ Most cases are due to a mutation in gene for rhodopsin or the gene for peripherin, a glycoprotein located in photoreceptor outer segment. ${ }^{35}$

Genes causing RP belong to different functional categories and their protein products function in several different processes. It is not possible within the scope of this review to discuss the functions of all RP genes. The known genes can be classified into metabolic groups according to the encoded protein: visual transduction, visual cycle, transcription factors, structural proteins, spliceosome complex, and cellular traffic, indicating the high level of specialisation of photoreceptors and of the retinal pigment epithelium. ${ }^{36}$ Some genes are specific to the retina or to photoreceptors (such as members of the phototransduction pathway, structural and transport proteins in photoreceptors, retina-specific transcription factors, and vitamin A metabolism); others have a more ubiquitous expression and function (for example, mRNA splicing); several genes encode proteins whose function in the retina is not well understood.

In parallel with this classification, genotype/ phenotype correlations have been established that will help ophthalmologists to suspect particular genes, and thereby mechanisms. This approach will provide better informations to patients and will orient the choice of future therapies. ${ }^{37}$ Genetic testing for RP patients is of potential value in clinical diagnosis and counselling. It has been sought in order to confirm the clinically established or suspected diagnosis, identify carrier status, and for predictive testing to detect the presence of a familial mutation in asymptomatic family members. Along with clinical correlates established for mutations in specific genes, genetic testing can be used for estimating the possible prognosis of disease. Knowledge of the types of mutations that occur and their relative (quantitative) importance is potentially of use in genetic counselling of families. Furthermore, in the event that a therapeutic approach becomes feasible in the future for a 
subset of RP patients having specified genetic defect(s), such knowledge can enable one to target the appropriate groups of patients for therapy. ${ }^{37}$ Although the basic pathophysiology of the disease is not exactly known, the occurrence of RP in IMDs suggests that it might be induced by abnormal metabolic products, errors of synthetic pathways, or deficient energy metabolism. ${ }^{38}$

A mutation in a copper-transporting ATP7A gene causes Menke's disease, characterised by low serum copper levels, low ceruloplasmin levels and retinal degeneration, mental retardation, hypopigmentation, and unusual hair ('kinky'). ${ }^{39}$ Patients with IMDs of lipid metabolism like Zellweger's syndrome (peroxisome biogenesis disorders), Refsum's disease (deficiency of phytanic acid oxidase) and mitochondrial $\beta$-oxidation defects exhibit retinal degeneration. Two rare forms of $\mathrm{RP}$, associated with the Bassen-Kornzweig's syndrome and Refsum's disease, respectively, yielded to treatment once the biochemical abnormalities were understood. ${ }^{33}$

Patients with Bassen-Kornzweig syndrome cannot efficiently transport fat-soluble vitamins from the intestine to the plasma. Treatment of a patient with large doses of vitamin A at an early stage resulted in reversal of the ERG to normal within $24 \mathrm{~h}$. Vitamin E also has been advocated to prevent progression of this retinal degeneration. Patients with Refsum's disease have an elevated serum phytanic acid resulting from a deficiency of phytanic acid oxidase. This fatty acid accumulates in the retinal pigment epithelium, leading to photoreceptor cell degeneration. Treatment with a low-phytol, lowphytanic acid diet has resulted in the lowering of serum phytanic acid and stabilisation of retinal function. ${ }^{33}$

Gyrate atrophy of the choroid and retina is a rare autosomal recessive disorder characterised by progressive metabolic, retinal, and choroidal degeneration due to photoreceptor degeneration caused by the deficiency of the pyridoxal phosphate-dependent, nuclear-encoded, mitochondrial matrix enzyme ornithine $\delta$-aminotransferase, which has been mapped to chromosome 10q26. ${ }^{40}$ Extreme hyperornithinemia is universal in this disease. Its absence necessitates a search for another diagnosis. All body fluids measured to date (whole blood, plasma, cerebrospinal fluid (CSF), aqueous humour, and urine) have been found to contain 10-20 times the normal levels of ornithine. As a result, excessive ornithine build-up causes the retinal thinning. Currently, this condition can only be treated with aminoacid tablets and a very low protein diet with limited fruits and vegetables and $>2000 \mathrm{cal}$ a day from carbohydrates and fats. Some patients cannot maintain this diet, and they need other treatment. One possible alternative is to replace the defective gene with one that functions normally, by gene therapy, which is currently under clinical trials. ${ }^{41}$
Neuronal ceroid lipofuscinoses (NCLs) is the general name for a family of at least eight genetically separate neurodegenerative disorders that result from excessive accumulation of lipopigments (lipofuscin) in the body's tissues. Juvenile NCL (JNCL, Batten disease), with a prevalence of 1 in 100000 , usually arises between 4 and 10 years of age; the first symptoms include considerable vision loss due to RP, with seizures, psychological degeneration, and eventual death in the mid- to late-20s ensuing. ${ }^{42,43}$ All mutations resulting in the juvenile variant of NCL have been shown to occur at the CLN3 gene on 16p12..4 The wild-type CLN3 gene codes for a protein with no known function ${ }^{45}$ however, studies of the yeast CLN3 ortholog, the product of which is called Battenin (after its apparent connections to Batten's disease, or JNCL), have suggested that the protein may have a role in lysosomal $\mathrm{pH}$ homeostasis.

To diagnose Batten's disease/NCL, the neurologist needs the patient's medical history and information from various laboratory tests. One of the tests is skin/tissue sampling under electron microscope. The powerful magnification of the microscope helps the doctor spot typical NCL deposits. These deposits are found in many different tissues, including the skin, muscle, conjunctiva, and others. These deposits take on characteristic shapes, depending on the variant under which they are said to occur; fingerprint profiles are typically found in JNCL. Electrical studies of the eyes, which include visualevoked responses and ERGs, can detect various eye problems common in childhood Batten's disease/NCLs. A recent development in diagnosis of Batten's disease/ NCL is the use of enzyme assays that look for specific missing lysosomal enzymes for infantile and late infantile disease only. This is a quick and easy diagnostic test. Currently, there is no widely accepted treatment that can cure, slow down, or halt the symptoms of NCL, other than supportive therapy. Gene therapy is under trial for patients with Batten's disease. NCL is currently under trial to test the effectiveness of bone marrow/neural stem cell transplants for this condition. ${ }^{46}$

The term 'cherry red spot' describes the ophthalmoscopic appearance of the retina in neurometabolic disorders such as Tay-Sach's disease, as described by Warren Tay. ${ }^{47}$ This fundus appearance also accompanies other neuronal lipid-storage disorders, including Sandhoff's disease (GM2 type II), gangliosidosis GM1 type I and GM2 type III, NiemannPick's disease, sialidosis types I and II, Farber's disease, mucolipidosis III, and metachromatic leukodystrophy (MLD) ${ }^{48-50}$ It often follows central retinal artery occlusion, which shows a pale retina as a result of reduced blood flow. The colour of the fovea, however, results from the pigment epithelium and choroid. The absence of ganglion cells at the fovea gives rise to red 
spot surrounded by white diseased cells. The various tones of normal pigmentation in the fovea, which lacks ganglion cells, contrast with the surrounding macular region of the retina, in which intracellular accumulation of metabolic products results in opacification during the neural disease process. Thus, the 'cherry red spot' could appropriately be renamed the 'perifoveal white patch.' Most patients with MPSs also exhibit macular cherry red spot. ${ }^{13,16}$ In MPSs, the abnormal metabolic products would be toxic to the retina. In other diseases, the pathogenesis of retinal involvement remains to be clarified. ${ }^{15}$

\section{IMDs involving the optic nerve}

Leber's hereditary optic neuropathy (LHON) or Leber's optic atrophy is a mitochondrially inherited degeneration of retinal ganglion cells (RGCs) and their axons that leads to an acute or subacute loss of central vision; this affects predominantly young adult males. ${ }^{51}$ Mitochondrial inheritance was first confirmed in 1988 with the identification of a mitochondrial DNA (mtDNA) point mutation at nucleotide position 11778 in the NADH dehydrogenase subunit 4 gene in nine pedigrees with a clinical diagnosis of LHON. ${ }^{52}$ A second mutation at nucleotide position 3460 was identified in three pedigrees without the 11778 mutation in $1991 .{ }^{53} \mathrm{LHON}$ is associated with three different point mutations of mtDNA affecting nucleotide positions 3460, 11778, and $14484 .^{51}$ These mutations are estimated to account for $8-25,50-60$, and $10 \%$ of LHON pedigrees, respectively. ${ }^{54}$ The incidence of each mutation is reported to be race dependent. ${ }^{55}$ Mitochondrial diseases affect most severely those tissues that have the greatest requirements for oxidative phosphorylation, such as the photoreceptors.

Costeff's optic atrophy syndrome or type III 3methylglutaconic aciduria, an IMD of leucine metabolism, is a neuro-ophthalmologic syndrome that consists of early onset bilateral optic atrophy and lateonset spasticity, extrapyramidal dysfunction, and cognitive deficit. Urinary excretion of 3-methylglutaconic acid and of 3-methylglutaric acid is increased. ${ }^{56}$ Krabbe's disease is an autosomal recessive sphingolipidosis caused by deficient activity of the lysosomal hydrolase galactosylceramide $\beta$-galactosidase due to mutation in the gene mapped to chromosome band $14 \mathrm{q} 31.3 .^{57}$ This enzyme degrades galactosylceramide, a major component of myelin, and other terminal $\beta$-galactosecontaining sphingolipids, including psychosine (galactosylsphingosine). Increased galactosylceramide and psychosine levels are believed to lead to widespread destruction of oligodendroglia in the CNS and to subsequent demyelination. Hallmarks of the classic infantile form are irritability, hypertonia, hyperaesthesia, and psychomotor arrest, followed by rapid deterioration, elevated protein levels in the CSF, neuro-radiologic evidence of white matter disease, cortical blindness, optic atrophy, and early death. ${ }^{57}$

Metachromatic leukodystrophy is a demyelinating storage disease caused by deficiency of the lysosomal enzyme arylsulphatase A, leading to the accumulation of galactosylceramide-3-o-sulphate (sulphatide) in the central and peripheral nervous systems. Patients with MLD show the storage of metachromatic complex lipids in the RGCs and in the optic nerve, leading to optic atrophy. ${ }^{58}$ The physiopathological process leading to neuronal cell degeneration and apoptosis in the optic nerve involves accumulation of undegraded sulphatides but also secondary abnormalities (storage/ mislocalisation of unrelated lipids, inflammatory processes). ${ }^{59}$

Gaucher's disease is a lysosomal storage disorder caused by a recessively inherited deficiency of glucocerebrosidase activity, which causes an accumulation of sphingolipid glucosylceramide in cells of the reticulo-endothelial systems. Ocular manifestations of Gaucher's disease include infiltration of the retina, conjunctiva, and uvea, with visual loss and eye movement disorders. ${ }^{23,60,61}$ Enzyme replacement treatment with intravenous recombinant glucocerebrosidase can dramatically result in dramatic improvement. ${ }^{62-67}$ Gene therapy may be a future step. Gaucher's disease has recently become a target for more than one effort at pharmacological chaperoning, which involves the use of orally administered drugs that operate at a molecular level. The currently existing treatment of Gaucher's disease, Cerezyme (imiglucerase for injection), is expensive and the treatment should be continued for life. ${ }^{65}$

\section{Screening and testing for IMDs}

For newborn screening, it is necessary to design, reliable screening tests (simple, inexpensive, with low falsenegative results) for IMDs (Tables 3 and 4). Accurate diagnosis is important for medical management, determining prognosis and genetic counselling. ${ }^{13,14}$ Testing and treatment for IMDs is often noninvasive and can be extended in an outpatient setting, provided it is ably supported by laboratory services. Simple screening tests may aid in diagnosis and evaluation of a suspected case of IMD and provide direction for more comprehensive laboratory analysis. ${ }^{68}$ In most cases, diagnosis can be established without any biopsy through biochemical analysis of blood and/or urine for specific metabolites. On special occasions, cerebrospinal and amniotic fluids (for estimation of amino acids, carnitine, organic acids, mucopolysaccharides, enzyme(s), and so 
Table 3 Initial laboratory screening for IMDs ${ }^{9,13,14,68,69}$

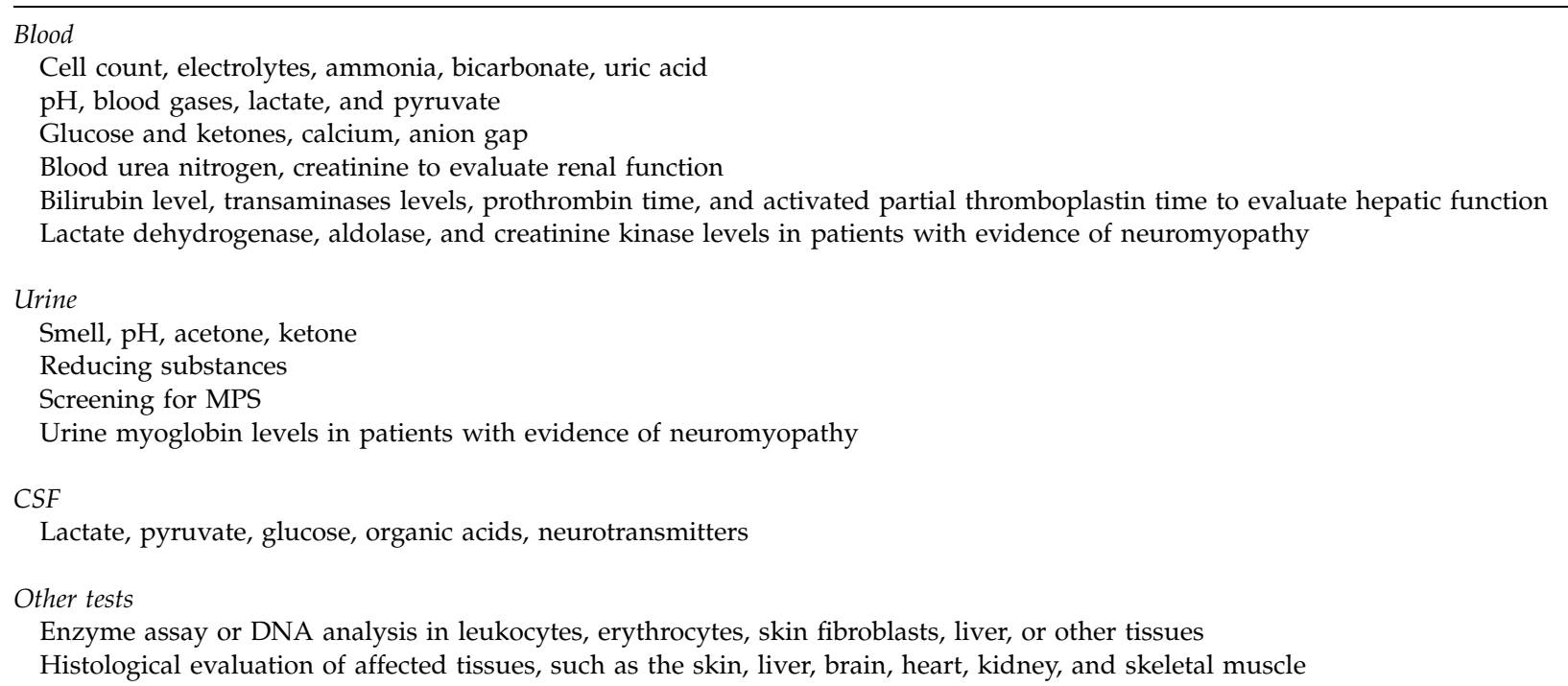

DNA, deoxyribonucleic acid; IMDs, inherited metabolic disorders; MPS, mucopolysaccharidoses.

Table 4 Specialised biochemical testing for IMDs ${ }^{9,13,14,68-70}$

Amino acid analysis in the blood, urine, or body fluids by thin layer chromatography (TLC) or high-performance liquid chromatography (HPLC).

Maple syrup urine disease (MSUD): increased branched-chain amino acids such as leucine, valine and isoleucine.

Hyperglycinemia: increased glycine.

Gyrate atrophy of retina: increased ornithine.

Costeff's optic atrophy: increased 3-methyl glutaconic acid.

Homocystinuria: increased homocysteine.

Phenylketonuria (PKU): increased phenylalanine metabolites.

Organic acidaemias: prenatal diagnosis in amniotic fluid for organic acids by gas liquid chromatography-mass spectrometry (GLC-MS) and acyl carnitine/carnitine by tandem mass spectrometry (MS).

Chromatography of glycolipids by TLC, GLC-MS and HPLC in IMDs of lipid metabolism.

Tandem MS for amino acids in the blood, urine, CSF, and tissues in MSUD, PKU, and homocystinuria.

GLC-MS for MSUD, multiple carboxylase deficiency, galactosaemia, ornithine transcarbomylase deficiency, tyrosinemia, etc.

Increased levels of long-chain fatty acids with peroxisomal disorder by TLC, HPLC, or GLC.

GLC-MS, gas liquid chromatography-mass spectrometry; HPLC, high-performance liquid chromatography; IMDs, inherited metabolic disorders; MSUD, maple syrup urine disease; PKU, phenylketonuria; tandem MS, tandem mass spectrometry; TLC, thin layer chromatography.

on) and chorionic villi are also used for diagnosis based on biochemical investigations.

In view of the nonspecific nature of clinical manifestations of IMDs, laboratory-based diagnosis often has a major role in confirmation or otherwise to rule out the suspected case of IMD. Confirmed diagnosis based on laboratory investigations helps in early medical intervention. As most of the laboratory investigations are often based on chemical analysis of metabolites or measuring the enzyme(s) activity, it is of importance to note that the specimen(s) for biochemical analysis needed to be collected at the right time, ideally during the crisis period. Further precautions need to be taken to transport the specimen in an ideal condition and also preserve it for suitable analytical purpose.

Through proper diagnosis and treatment, it is possible to prevent the natural history of the disease. ${ }^{69}$ The increasing application of new technologies, such as electrospray ionisation tandem mass spectrometry, ${ }^{70}$ to newborn screening in asymptomatic persons allows 
earlier identification of IMDs. It also detects some conditions of uncertain clinical significance. ${ }^{68}$ Nuclear magnetic resonance spectroscopy can provide a noninvasive in vivo evaluation of proton-containing metabolites and can lead to diagnosis of certain rare, but potentially treatable, neurometabolic disorders. ${ }^{71}$ Electron microscopic evaluation of a skin biopsy is a highly sensitive screening tool that provides valuable clues to stored membrane material or ultrastructural organelle changes. ${ }^{72}$

\section{Recent advances in therapy}

\section{ERT}

Enzyme-replacement therapy follows the observation in 1970s that many lysosomal enzymes can be secreted and then sequestrated by lysosomes in distant tissues. Mannose-6-phosphate receptors present on numerous cell membranes bind lysosomal enzymes with mannose6-phosphate residues and facilitate the uptake of lysosomal enzymes. ${ }^{73}$ Early tissue culture experiments showed that exogenous enzymes could gain access to and degrade the accumulated intracellular substrates. ${ }^{74,75}$ Even with achievement of 1-5\% of normal cellular activity after supplying exogenous enzymes, these in vitro studies showed that storage substances dwindled. After these early exciting discoveries in 1970s, the progress in the 1980s was relatively slow. It was because of the difficulty of manufacturing large quantities of purified lysosomal enzymes and the lack of animal models of some human lysosomal storage disorders (LSDs).

The first enzyme available for treating LSDs was alglucerase for Gaucher's disease. In 1990s, advances in clinical genetics sped up the development of ERT. First, by means of genetic engineering, production of large quantities of recombinant enzymes became feasible. Second, knockout mouse models for LSDs became technologically possible providing animal models of LSDs for preclinical trials. ${ }^{76}$ Hence, since the successful production of imiglucerase by recombinant technology in 1996 replacing alglucerase, several other lysosomal enzymes produced by recombinant DNA technology were studied in preclinical and clinical trials. Enzymes developed for treatment of Fabry's disease and mucopolysaccharidosis type I were approved in Europe and the United States in the early 2000s. Currently, clinical trials of ERT are going on for many other types of MPSs. ${ }^{76-79}$

The study of pharmacokinetics and pharmacodynamics of exogenous enzymes for treatment of Gaucher's disease deepened the understanding of how exogenous enzymes work in the body. Exogenous enzymes once given intravenously are rapidly taken up into cells resulting in a short serum half-life of around 10-20 min. However, exogenous enzymes are not uniformly taken up. The efficient and preferential uptake of exogenous enzymes into certain compartments of the bodies leads to rapid clearance of enzymes in the bloodstream and deprives the availability of enzymes for uptake into less-accessible compartments. ERT has always enjoyed good reputation for its safety. Infusionrelated reactions, such as urticarial rash, chills and rigors, and headache are common but not serious. It is because of the development of antibodies against the exogenous enzymes. Slowing infusion rate lessens the severity of such reactions. Fortunately, other than causing infusionrelated reactions, antibodies developing during the course of ERT are rarely neutralising and effectiveness of ERT is usually not hampered as a result. It was found that development of antibodies is correlated with the residual enzyme activities in patients. One intrinsic problem of ERT is that ERT has so far not been effective in the treatment of neurological manifestations of LSDs, as exogenous enzymes cannot penetrate the blood-brain barrier. Thus, application and development of other treatment modalities remains important. ${ }^{80}$

\section{Enzyme-enhancement therapy}

In some LSDs, mutations cause misfolding of enzyme protein and thus impairing transport of enzymes into the lysosomes from the endoplasmic reticulum. Chaperones are low molecular weight molecules that help unfold the proteins and thus enhance the residual enzyme activity. On the basis of this principle, a patient with the cardiac variant of Fabry's disease who had severe heart complications was treated with galactose infusions $(1 \mathrm{~g} / \mathrm{kg})$ three times weekly. ${ }^{81}$ There was marked clinical improvement obviating cardiac transplantation. This is an area with great potential for development. As chaperones are small enough to cross the blood-brain barrier, there is hope that neurological manifestations of LSDs could be effectively treated by chaperones and this awaits confirmation by clinical studies.

\section{Substrate-deprivation therapy}

Substrate-deprivation therapy makes use of small molecules that inhibit synthesis of storage substances in LSDs. The inhibition of synthesis of storage substances coupled with the remaining enzyme activities results in the gradual disappearance of storage substances in cells. Theoretically, these small molecules can be taken up into the CNS and potentially can treat LSDs with involvement of the CNS. N-butyl-deoxynojirimycin is the first of such small molecules, which inhibits ceramide-specific 
glucosyltransferase preventing the formation of glucocerebroside, the storage compound in Gaucher's disease. $^{82}$

\section{Conclusion}

Recognition of IMD as cause of eye disease has implications both for care of the patient and for genetic counselling or prenatal diagnosis. Some diseases are amenable to symptomatic treatment. In critically ill infant, aggressive treatment before definitive confirmation of diagnosis is lifesaving and may reduce long-term sequelae. Metabolic acidosis should be aggressively treated with sodium bicarbonate. Seizures in infancy are treated with anti-epileptic drugs or pyridoxine. Traditional therapies for IMDs include dietary therapy, such as protein restriction, cofactor supplements, and so on. Evolving therapies include organ transplantation and enzyme replacement. Efforts to provide treatment through somatic gene therapy are in early stage, but there is hope that this approach will provide additional therapeutic possibilities. A detailed ophthalmological assessment is mandatory. A combined approach and management by an ophthalmologist, paediatrician, biochemist, and medical geneticist is warranted in most cases. Metabolic investigation(s) should be preceded by an initial clinical and biochemical workup after which the biochemist specialised in metabolic diseases performs the laboratory tests for definitive diagnosis of IMDs. Recent advances in diagnosis and treatment have significantly improved the prognosis for many infants with inborn errors of metabolism.

\section{References}

1 Garrod AE. Inborn errors of metabolism. In: Frowde H Hodder and Stoughton (eds). The Croonian Lectures Delivered Before the Royal College of Physicians, London, June 1908, 2nd edn. Oxford University Press: London, 1909.

2 Childs B. Sir Archibald Garrod's conception of chemical individuality: a modern appreciation. N Engl J Med 1970; 282: 71-77.

3 Beaudet AL, Scriver CR, Sly WS, Valle D. Molecular basis of variant human phenotypes. In: Scriver CR (ed). The Metabolic and Molecular Bases of Inherited Disease, 8th edn. McGraw-Hill: New York, 2001, pp 3-51.

4 Applegarth DA, Toone JR, Lowry RB. Incidence of inborn errors of metabolism in British Columbia, 1969-1996. Pediatrics 2000; 105: e10.

5 Meikle PJ, Hopwood JJ, Clague AE, Carey WF. Prevalence of lysosomal storage disorders. JAMA 1999; 281: 249-254.

6 Verma IC. Burden of genetic disease in India. Indian J Pediatr 2000; 67: 893-898.

7 ICMR Collaborating Centers and Centers co-ordinating unit. Multicentric study on genetic causes of mental retardation in India. Indian J Med Res 1991; 94: 161-169.
8 Waisbren SE, Albers S, Amato S, Ampola M, Brewster TG, Demmer L et al. Effect of expanded newborn screening for biochemical genetic disorders on child outcome and parental stress. JAMA 2003; 290: 2564-2572.

9 Kumta NB. Inborn errors of metabolism - an Indian perspective. Indian J Pediatr 2005; 72: 325-332.

10 Costa T, Scriver CR, Childs B. The effect of Mendelian disease on human health: a measurement. Am J Med Genet 1985; 21: 231-242.

11 Freund C, Horsford DJ, McInnes RR. Transcription factor genes and the developing eye: a genetic perspective. Hum Mol Genet 1996; 5: 1471-1488.

12 van Heyningen V. Developmental eye disease-a genome era paradigm. Clin Genet 1998; 54: 272-282.

13 Clarke JT. A Clinical Guide to Inherited Metabolic Diseases, 2nd edn. Cambridge University Press: New York, 2002.

14 Blan N, Duran M, Blaskovics ME, Gibson KM. Physician's Guide to the Laboratory Diagnosis of Metabolic Diseases, 2nd edn. Springer: New York, 2003.

15 Poll-The BT, Maillette de Buy Wenniger-Prick LJ, Barth PG, Duran M. The eye as a window to inborn errors of metabolism. J Inherit Metab Dis 2003; 26: 229-244.

16 Ashworth JL, Biswas S, Wraith E, Lloyd IC. Mucopolysaccharidoses and the eye. Surv Ophthalmol 2006; 51: 1-17.

17 Baehner F, Kampmann C, Whybra C, Miebach E, Wiethoff $\mathrm{CM}$, Beck M. Enzyme replacement therapy in heterozygous females with Fabry's disease: results of a phase IIIB study. J Inherit Metab Dis 2003; 26: 617-627.

18 Liu M, Cohen EJ, Brewer GJ, Laibson PR. Kayser-Fleischer ring as the presenting sign of Wilson's disease. Am J Ophthalmol 2002; 133: 832-834.

19 Ala A, Walker AP, Ashkan K, Dooley JS, Schilsky ML. Wilson's disease. Lancet 2007; 369: 397-408.

20 Roberts EA, Schilsky ML. A practice guideline on Wilson's disease. Hepatology 2003; 37: 1475-1492.

21 Walshe JM. Treatment of Wilson's disease: the historical background. QJM 1996; 89: 553-555.

22 Tsilou E, Zhou M, Gahl W, Sieving PC, Chan CC. Ophthalmic manifestations and histopathology of infantile nephropathic cystinosis: report of a case and review of the literature. Surv Ophthalmol 2007; 52: 97-105.

23 Iwata F, Kaiser-Kupfer MI. Ocular manifestations of metabolic disorders. Curr Opin Ophthalmol 1994; 5: 79-83.

24 Lee AYW, Chung SK, Chung SSM. Demonstration that polyol accumulation is responsible for diabetic cataract by the use of transgenic mice expressing the aldose reductase gene in the lens. Proc Natl Acad Sci USA 1995; 92: 2780-2784.

25 Cali JJ, Hsieh CL, Francke U, Russell DW. Mutations in the bile acid biosynthetic enzyme sterol 27-hydroxylase underlie cerebrotendinous xanthomatosis. J Biol Chem 1991; 266: 7779-7783.

26 Salen G, Shefer S, Tint GS. Transformation of 4-cholesten-3one and 7-hydroxy-4-cholesten-3-one into cholestanol and bile acids in cerebrotendinous xanthomatosis. Gastroenterol 1984; 87: 276-283.

27 Koopman BJ, Wolthers BG, van der Molen JC, van der Slik W, Waterreus RJ, van Spreeken A. Cerebrotendinous xanthomatosis: a review of biochemical findings of the patient population in the Netherlands. J Inherit Metab Dis 1988; 11: 56-75.

28 Kuriyama M, Tokimura Y, Fujiyama J, Utatsu Y, Osame M. Treatment of cerebrotendinous xanthomatosis: effects of 
chenodeoxycholic acid, pravastatin, and combined use. J Neurol Sci 1994; 125: 22-28.

29 Traboulsi EI, Whittum-Hudson JA, Mir SH, Maumenee IH. Microfibril abnormalities of the lens capsule in patients with Marfan's syndrome and ectopia lentis. Ophthalmic Genet 2000; 21: 9-15.

30 Mudd SH. Disorders of transulfuration. In: Scriver CR (ed). The Metabolic and Molecular Bases of Inherited Disease, 8th edn. McGraw-Hill: New York, 2001, pp 2007-2056.

31 Lueder GT, Steiner RD. Ophthalmic abnormalities in molybdenum cofactor deficiency and isolated sulfate oxidase deficiency. J Pediatr Ophthalmol Strabismus 1995; 32: 334-337.

32 Rattner A, Sun H, Nathans J. Molecular genetics of human retinal disease. Annu Rev Genet 1999; 33: 89-131.

33 Berson EL. Retinitis pigmentosa. Invest Ophthalmol Vis Sci 1993; 34: 1656-1676.

34 Maubaret C, Hamel C. Genetics of retinitis pigmentosa: metabolic classification and phenotype/genotype correlations. J Fr Ophtalmol 2005; 28: 71-92.

35 Drija TP. Retinitis pigmentosa and stationary night blindness. In: Scriver CR (ed). The Metabolic and Molecular Bases of Inherited Disease, 8th edn. McGraw-Hill: New York, 2001, pp 5903-5933.

36 Rozet JM, Perrault I, Gigarel N, Souied E, Ghazi I, Gerber S et al. Dominant $\mathrm{X}$ linked retinitis pigmentosa is frequently accounted for by truncating mutations in exon ORF15 of the RPGR gene. J Med Genet 2002; 39: 284-285.

37 Kannabiran C. Retinitis pigmentosa: genetics and gene based approaches to therapy. Expert Rev Ophthalmol 2008; 3 417-429.

38 Bhatti MT. Retinitis pigmentosa, pigmentary retinopathies and neurologic diseases. Curr Neurol Neurosci Rep 2006; 6: 403-413.

39 Aldecoa V, Escofet-Soteras C, Artuch R, Ormazabal A, Gabau-Vila E, Martin-Martinez C. Menke's disease: its clinical, biochemical and molecular diagnosis. Rev Neurol 2008; 46: 446-447.

40 Wang T, Milan AH, Steel G, Valle D. A mouse model of gyrate atrophy of the choroid and retina: early pigment epithelium damage and progressive retinal degeneration. J Clin Invest 1996; 97: 2753-2762.

41 Weleber RG, Kurz DE, Trzupek KM. Treatment of retinal and choroidal degenerations and dystrophies: current status and prospects for gene based therapy. Ophthalmol Clin North Am 2003; 16: 583-593.

42 Persaud-Sawin DNW, van Dongen A, Boustany RN. Motifs within the CLN3 protein: modulation of cell growth rates and apoptosis. Hum Mol Gen 2002; 11: 2129-2142.

43 Haltia M. The neuronal ceroid-lipofuscinoses: from past to present. Biochim Biophys Acta 2006; 1762: 850-856.

44 Sharp JD, Wheeler RB, Lake BD, Savukoski M, Järvelä IE, Peltonen $\mathrm{L}$ et al. Loci for classical and a variant late infantile neuronal ceroid lipofuscinosis map to chromosomes 11p15 and 15q21-23. Hum Mol Genet 1997; 6: 591-595.

45 Narayan SB, Pastor JV, Mitchison HM, Bennett MJ. CLN3L, a novel protein related to the Batten disease protein, is overexpressed in $\mathrm{Cln} 3-/-$ mice and in Batten disease. Brain 2004; 127: 1748-1754.

46 Lonnqvist T, Vanhanen SL, Vettenranta K, Autti T, Rapola J. Hematopoietic stem cell transplantation in infantile neuronal ceroid lipofuscinosis. Neurology 2001; 57: 1411-1416.

47 Tay W. Symmetrical changes in the region of the yellow spot in each eye of an infant. Trans Ophthalmol Soc UK 1881; 1: 55-57.
48 Kivlin JD, Sanborn GE, Myers GG. The cherry-red spot in Tay-Sach's and other storage diseases. Ann Neurol 1985; 17: 356-360.

49 Taylor D. Neurometabolic disease. In: Taylor D (ed). Pediatric Ophthalmology, 2nd edn. Blackwell Science Ltd: Boston, 1997, pp 772-793.

50 Guseva MR, Pavliuk EIU. The cherry red spot is an early sign of Farber's lipogranulomatosis. Vestn Oftalmol 2008; 124: 51-53.

51 Man PYW, Turnbull DM, Chinnery PF. Leber hereditary optic neuropathy. J Med Genet 2002; 39: 162-169.

52 Wallace DC, Singh G, Lott MT, Hodge JA, Schurr TG, Lezza AM et al. Mitochondrial DNA mutation associated with Leber's hereditary optic neuropathy. Science 1988; 242: 1427-1430.

53 Huoponen K, Vilkki J, Aula P, Nikoskelainen EK, Savontaus ML. A new mitochondrial DNA mutation associated with Leber hereditary optic neuroretinopathy. Am J Hum Genet 1991; 48: 1147-1153.

54 Hwang JM, Chang BL, Koh HJ, Kim JY, Park SS. Leber's hereditary optic neuropathy with 3460 mitochondrial DNA mutation. J Korean Med Sci 2002; 17: 283-286.

55 Howell N, Halvorson S, Burns J. When does bilateral optic atrophy become Leber hereditary optic neuropathy? Am J Hum Genet 1993; 53: 959-963.

56 Anikster Y, Kleta R, Shaag A, Gahl WA, Elpeleg O. Type III 3-methyl glutaconic aciduria (optic atrophy plus syndrome or Costeff optic atrophy syndrome): identification of the OPA3 gene and its founder mutation in Iraqi Jews. Am J Hum Genet 2001; 69: 1218-1224.

57 Suzuki K. Globoid cell leukodystrophy (Krabbe's disease): update. J Child Neurol 2003; 18: 595-603.

58 Gieselmann V. Metachromatic leukodystrophy: genetics, pathogenesis and therapeutic options. Acta Paediatr Suppl 2008; 97: 15-21.

59 Sevin C, Aubourg P, Cartier N. Enzyme, cell and gene based therapies for metachromatic leukodystrophy. J Inherit Metab Dis 2007; 30: 175-183.

60 Petrohelos M, Tricoulis D, Kotsiras I, Vouzoukos A. Ocular manifestations of Gaucher's disease. Am J Ophthalmol 1975; 80: 1006-1010.

61 Morales LE. Gaucher's disease: a review. Ann Pharmacother 1996; 30: 381-388.

62 Grabowski GA. Phenotype, diagnosis, and treatment of Gaucher's disease. Lancet 2008; 372: 1263-1271.

63 Brunel-Guitton C, Rivard GE, Galipeau J, Alos N, Miron $\mathrm{MC}$, Therrien $\mathrm{R}$ et al. Enzyme replacement therapy in pediatric patients with Gaucher's disease: what should we use as maintenance dosage? Mol Genet Metab 2009; 96: 73-76.

64 Charrow J. Enzyme replacement therapy for Gaucher's disease. Expert Opin Biol Ther 2009; 9: 121-131.

65 Weinreb NJ. Imiglucerase and its use for the treatment of Gaucher's disease. Expert Opin Pharmacother 2008; 9: 1987-2000.

66 Connock M, Burls A, Frew E, Fry-Smith A, Juarez-Garcia A, McCabe $C$ et al. The clinical effectiveness and costeffectiveness of enzyme replacement therapy for Gaucher's disease: a systematic review. Health Technol Assess 2006; 10: iii-iv, ix-136.

67 Krivit W. Allogeneic stem cell transplantation for the treatment of lysosomal and peroxisomal metabolic diseases. Springer Semin Immunopathol 2004; 26: 119-132.

68 Holtzman NA. Expanded newborn screening: how good is the evidence? JAMA 2003; 290: 2606-2608. 
69 Raghuveer TS, Garg U, Graf WD. Inborn errors of metabolism in infancy and early childhood: an update. Am Fam Physician 2006; 73: 1981-1990.

70 Wilcken B, Wiley V, Hammond J, Carpenter K. Screening newborns for inborn errors of metabolism by tandem mass spectrometry. N Engl J Med 2003; 348: 2304-2312.

71 Novotny E, Ashwal S, Shevell M. Proton magnetic resonance spectroscopy: an emerging technology in pediatric neurology research. Pediatr Res 1998; 44: 1-10.

72 Prasad A, Kaye EM, Alroy J. Electron microscopic examination of skin biopsy as a cost effective tool in the diagnosis of lysosomal storage diseases. J Child Neurol 1996; 11: 301-308.

73 Kornfeld S. Lysosomal enzyme targeting. Biochem Soc Trans 1990; 18: 367-374.

74 O'Brien JS, Miller AL, Loverde AW, Veath ML. Sanfilippo disease type B: enzyme replacement and metabolic correction in cultured fibroblasts. Science 1973; 181: 753-755.

75 Cantz M, Kresse H. Sandhoff disease: defective glycosaminoglycan catabolism in cultured fibroblasts and its correction by beta-N-acetylhexosaminidase. Eur J Biochem 1974; 47: 581-590.

76 Muenzer J, Towle D, Calikoglu M, McCandless S. A phase I/ II clinical study evaluating the safety and clinical activity of enzyme replacement therapy in mucopolysaccharidosis II (Hunder syndrome). Am J Hum Genet 2002; 71 (Suppl 4): 582.
77 Harmatz P, Whitley CB, Waber L, Pais R, Steiner R, Plecko B et al. Enzyme replacement therapy in mucopolysaccharidosis VI (Maroteaux-Lamy syndrome). J Pediatr 2004; 144: 574-580.

78 van den Hout JM, Reuser AJ, de Klerk JB, Arts WF, Smeitink JA, Van der Ploeg AT. Enzyme therapy for Pompe's disease with recombinant human $\alpha$-glucosidase from rabbit milk. J Inherit Metab Dis 2001; 24: 266-274.

79 Amalfitano A, Bengur AR, Morse RP, Majure JM, Case LE, Veerling DL et al. Recombinant human acid $\alpha$-glucosidase enzyme therapy for infantile glycogen storage disease type II: results of a phase I/II clinical trial. Genet Med 2001; 3: $132-138$.

80 Desnick RJ, Schuchman EH. Enzyme replacement and enhancement therapies: lessons from lysosomal disorders. Nat Rev Genet 2002; 3: 954-966.

81 Frustaci A, Chimenti C, Ricci R, Natale L, Russo MA, Pieroni $\mathrm{M}$ et al. Improvement in cardiac function in the cardiac variant of Fabry's disease with galactose infusion therapy. N Engl J Med 2001; 345: 25-32.

82 Pastores GM, Barnett NL. Substrate reduction therapy: miglustat as a remedy for symptomatic patients with Gaucher's disease type I. Expert Opin Investig Drugs 2003; 12: $273-281$. 